\title{
A new approach of the concept of prime number
}

\author{
Jamel Ghanouchi
}

Professor of Mathematics

RIME Department of Mathematics

6 Rue Khansa 2070 Marsa Tunisia

\section{Keywords : Reals ; Primes ; Transcendentality ; Cryptology}

\section{Abstract}

In this paper, we generalize the concept of prime number and define the real pr hes. It allo to apply the new concept to cryptology.

\section{Introduction}

The prime numbers are called primes because they are the bricks $\prod_{j} p_{j}^{n_{j}}$ when where $p_{j}$ are primes and $n$
can be written as
decomposition in prime factors of the number $\mathrm{n}$. In fact, th decomposition in prime factors of the number $\mathrm{n}$. In fact, th finition particular case of a
much more general one. Indeed, if $n_{j}$ are rationals, everything chan cs. Considering that the decomposition in prime factors of an integer $\mathrm{n}$ when $j$
the $j p$ have no reason to be the same than before and theyalon $\prod_{j} p_{j}^{n_{j}}$. In this writhing, then decide that 16 is conventionally prime, we hat nul to 16 power $1 / 4$ and each number can be written according to 16 and its rational exponent nste

If we decide conventionally that each Eermat nu, wer is prime, and it is possible by the fact that they are coprime two by two, then ach w prim (new primes=bricks with rational exponents in the writing) replaces anther on the ist of th old primes (old primes=bricks with integral exponents in the writing).

Example : If by convention the fifith nat number $=4294967297=641.6700417$ is prime, we can decide that it replaces 6 becom, compound and 6700417 is prime or 641 is prime and 67004147 is compourd

In all cases, the ad vantage is that have a formula which gives for each $n$ a prime. And we can see the the prip are sinite. There is another interesting result : Let Ulam spiral. The Fermat numbers are all s, $d$ in the ame line.

\section{DeI}

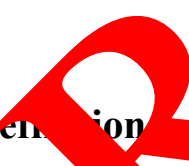

A real nuts $\prod p_{j}{ }^{{ }_{j}}$ $\mathrm{r}$ is compound if it can be written as ${ }_{j}$ where $p_{j}$ are primes and $\mathrm{j} \mathrm{n}$ are rationals. This ecomposition in prime factors is unique. A prime real number or R-prime can be written only as $\mathrm{p}=\mathrm{p} .1$. Thus we define other real prime numbers like $\pi, \mathrm{e}, \ln (2)$. Of course, it is a convention, because, we can consider $\pi^{2}$ as prime and $\pi$ will be no more prime. It is equivalent in what will follow.

Thus $\sqrt[q]{p}=p^{\frac{1}{q}}$ is compound. Also we have $\sqrt[q]{p}+1=p^{\frac{1}{q}}+1$ when $\mathrm{p}$ is prime and we have $\sqrt[2^{i}]{p}-1=(p-1)(\sqrt[2]{p}+1)^{-1}(\sqrt[i-1]{p}+1)^{-1} \ldots(\sqrt{p}+1)^{-1}$ compound for $\mathrm{p}$ prime, for example.

Another example : $\sqrt[3]{p^{2}}-\sqrt[3]{p}+1=(p+1)(\sqrt[3]{p}+1)^{-1}$

It is $5 / 2$ that divises 5 not the contrary! 
2. Division of a real by a real

The GCD of two numbers

$\mathrm{p}$ and $\mathrm{q}$ are prime numbers :

$p \neq q \Rightarrow G C D(p, q)=1$

$n m<0 \Rightarrow G C D\left(p^{n}, p^{m}\right)=1$

$m n>0 ; m>0 ; G C D\left(p^{n}, p^{m}\right)=p^{\min (m, n)}$

$m n<0 ; m<0 ; G C D\left(p^{n}, p^{m}\right)=p^{\max (m, n)}$

$i \geq n_{l} \geq 1 ; G C D\left(\prod_{n=1}^{n=i} p_{n}^{m_{n}}, \prod_{l=1}^{l=j} p_{n_{l}}^{{ }^{q_{n l}}}\right)=\prod_{l=1}^{l=j} G C D\left(p_{n_{l}}^{{ }^{m_{n l}}}, p_{n_{l}}{ }^{q_{n_{l}}}\right)$

So a real number $\mathrm{y}$ divises a real number $\mathrm{x}$ if $\operatorname{GCD}(\mathrm{x}, \mathrm{y})=\mathrm{y}$.

\section{Theorem}

$\mathrm{p}$ is prime then

$\forall a \in R, \exists k \in R ; a^{p}=a+k p$

4. Proof of the theorem

$$
\begin{aligned}
& a=\sum_{m=0}^{m=\infty} a_{m} \cdot 10^{u-m} ; a_{m} \in N \\
& \exists k, k^{\prime} ; a^{p}=\sum_{m=0}^{m=\infty} a_{m}{ }^{p} \cdot 10^{u-m}+k p=\sum_{m=\infty}^{m=0}\left(a_{m}+k\right)+k p=\sum_{m=0}^{m=\infty} a_{m} \cdot 10^{u-m}+k^{\prime \prime} p=a+k^{\prime \prime} p
\end{aligned}
$$

\section{The probabilities}

What the probability that a ber betwo $x+d x$ and $x$ is prime ? It is

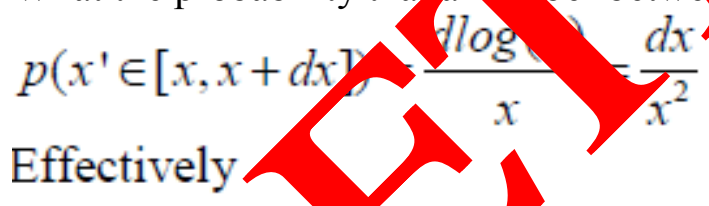

$\log \left(1+\frac{d x}{x} \log (x)-\log (x)=\frac{d x}{x}=d \log (x)\right.$

$p\left(x^{\prime} \in[x, y]\right)=p\left(x^{\prime} \in[0, x+d x]\right)-p\left(x^{\prime} \in[0, x]\right)=\frac{\log (x+d x)}{x+d x}-\frac{\log (x)}{x}$

$=\frac{\log (x+d x)}{x}-\frac{\log (x)}{x}=\frac{d \log (x)}{x}$

How many primes are there beyween $\mathrm{x}$ and $\mathrm{x}+\mathrm{dx}$ ? There are

$\pi(x)=\int \frac{d x}{d \log (x)}=\infty$ 


\section{Applications to cryptology}

Let us build real numbers $\mathrm{P}$ and $\mathrm{Q}$. We have $p_{l}$ a prime and $u_{n}$ a sequence.

We know that $p_{n}=1+\sqrt[u_{n}]{p_{n-1}}$ is a real. With $\mathrm{N}$ enough great, $P=p_{N}$. Also with another prime $q_{1}$ and another sequence $v_{n}$, we have another real with $\mathrm{M}$ enough great, $Q=q_{M}$. As

$1+\sqrt{P}$ is real and $1+\sqrt{Q}$ is real, let $n=\sqrt{P}+\sqrt{Q}$. Let $e=\alpha+u \sqrt{P}+v \sqrt{Q}$ and let $\mathrm{d}=\mathrm{kn}-\mathrm{e}$, If we have $\mathrm{n}$ and e public keys, the message is $\mathrm{M}=\mathrm{C}+\mathrm{e}+\mathrm{kn}$ and the cypher is $\mathrm{C}=\mathrm{M}-\mathrm{e}+\mathrm{k}$ 'n $=\mathrm{M}+\mathrm{d}+\mathrm{k}$ 'n.

Another possibility is to take $\mathrm{n}=\mathrm{PQ}$ and $e=\frac{\alpha}{(P-1)^{u}(Q-1)^{v}}$ then

$\mathrm{n}$ and $\mathrm{e}$ are the public keys and $M=C^{e}+k n$ then $C=M^{d}+k^{\prime} n$ with $d$

Example:

$p=79, q=83$

$p_{1}=\sqrt[5]{79}+1=3.39621299$

$q_{1}=\sqrt[7]{83}+1=2.879983394$

$P=\sqrt{p_{1}}+1=2.842881708$

$Q=\sqrt{q_{1}}+1=2.697051392$

$n=P Q=7.629465043$

$e=\frac{9378.2}{(P-1)^{7}(Q-1)^{6}}=5.737752231 \equiv 2.6341 .56((P,-1)(Q-1))$

$M=79.836$

$M^{e}=79.863^{2.63415566} \equiv 4.6$

$d=\frac{(P-1)^{7}(Q-1)^{8}}{\alpha}=3.3286$

$13445 \times 7.62946=043+4.6268 \quad \gamma 2580.2768$

$C=102580.2 \quad 0.3796 \Rightarrow 79.86373604 \Rightarrow C=79.863$

\section{Con usio}

We have neranzed the concept of prime to the reals. It allowed to present an application tocryptology

\section{References}

[1]. R. J. Backlund, « Sur les zéros de la fonction $\zeta(\mathrm{s})$ de Riemann », CRAS, vol. 158, 1914, p. 1979-1981.

[2]. X. Gourdon, « The 1013 first zeros of the Riemann zeta function, and zeros computation at very large height »

[3]. J.P.Gram, « Note sur les zéros de la fonction $\zeta($ s) de Riemann », Acta Mathematica, vol. 27, 1903, p. 289-304. 
[4]. J.I.hutchinson « On the Roots of the Riemann Zeta-Function », Trans. AMS, vol. 27, no 1, 1925, p. 49-60.

[5]. A. M. Odlyzko, The 1020-th zero of the Riemann zeta function and 175 million of its neighbors, 1992.

[6]. J.Barkley Rosser, J. M. Yohe et Lowell Schoenfeld, « Rigorous computation and the zeros of the Riemann zeta-function.», Information Processing 68 (Proc. IFIP Congress, Edinburgh, 1968), Vol. 1: Mathematics, Software, Amsterdam, North-Holland, 1969, p. 70-76.

[7]. http://fr.wikipedia.org/wiki/Edward_Charles_Titchmarsh E.C.Titchmarsh, « The Zeros of the Riemann Zeta-Function ", Proceedings of the Royal Society, Series A, Mathematical and Physical Sciences, vol. 151, no 873, 1935, p. 234-255.

[8]. E. C. Titchmarsh, "The Zeros of the Riemann Zeta-Function », Proceedings of tho Royal Society, Series A, Mathematical and Physical Sciences, The Royal Society, vol. 157, no 85, 936, p. 261-263.

[9]. A.M.Turing, «Some calculations of the Riemann zeta-function », Proceding of the I MS, Third Series, vol. 3, 1953, p. 99-117.

[10]. J. van de Lune, H.te Riele et D. T. Winter, « On the zeros of the R vann fund on in the critical strip. IV », Mathematics of Computation, vol. 46, no 174, 198 p. 081.

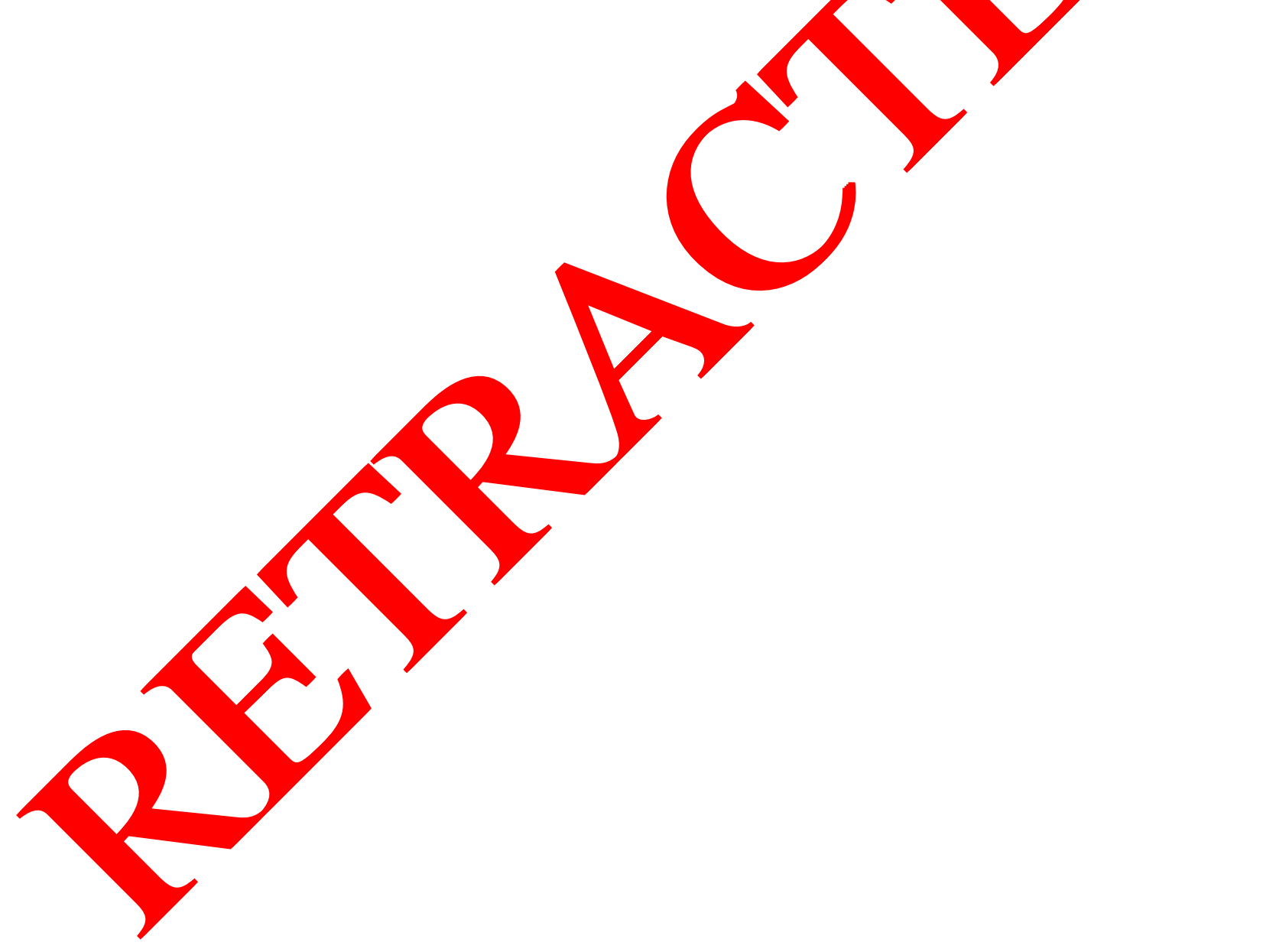

